\title{
Rician $K$-Factor-Based Analysis of XLOS Service Probability in 5G Outdoor Ultra-Dense Networks
}

\author{
Hatim Chergui, Member, IEEE, Mustapha Benjillali, Senior Member, IEEE, \\ and Mohamed-Slim Alouini, Fellow, IEEE
}

\begin{abstract}
In this letter, we introduce the concept of Rician $K$ factor-based radio resource and mobility management for fifth generation (5G) ultra-dense networks (UDN), where the information on the gradual visibility between the new radio node $B$ (gNB) and the user equipment (UE)-dubbed X-line-of-sight (XLOS)would be required. We therefore start by presenting the XLOS service probability as a new performance indicator; taking into account both the UE serving and neighbor cells. By relying on a lognormal $K$-factor model, a parametric expression of the XLOS service probability in a 5G outdoor UDN is derived, where the link between network parameters and the availability of a XLOS condition is established. The obtained formula is given in terms of the multivariate Fox H-function, wherefore we develop a fast graphical processing unit (GPU)-enebled MATLAB code. Residue theory is then applied to infer the relevant asymptotic behavior and show its practical implications. Finally, numerical results are provided for various network configurations, and underpinned by extensive Monte-Carlo simulations.
\end{abstract}

Index Terms-5G, GPU, multivariate Fox H-function, Rician $K$ factor, UDN, XLOS service probability.

\section{INTRODUCTION}

$\mathbf{T}$ HE emergence of 5G ultra-dense networks [1] will certainly prompt the reshaping of radio resource and mobility management algorithms, wherefore a new set of measured quantities might be required as inputs. In this context, the Rician $K$-factor can serve as an accurate channel metric to measure the gradual visibility condition of a radio link, termed $X$ line-of-sight (XLOS) here, and encompassing LOS, obstructedLOS (OLOS) and non-LOS (NLOS) as discrete regimes, where generally $K_{\mathrm{NLOS}} \ll K_{\mathrm{OLOS}}<K_{\mathrm{LOS}}$ [2]. In localization services for instance, while the availability of a LOS path is quintessential for the classical triangulation-based schemes such as time-of-arrival (TOA) and direction-of-arrival (DOA), the massive multiple-input multiple-output (MIMO)-based spacetime processing approaches can deliver very concise localization thanks to the high angular resolution of the large scale antennas, and may therefore operate in the worst OLOS/NLOS conditions, yet at the expense of a higher complexity [3]. To optimize the computational cost, an operator may adopt a hybrid network configuration where, according to a fine-tuned target $K$-factor threshold, the $5 \mathrm{G} \mathrm{gNB}$ can switch between the simpler conventional methods and the massive-MIMO ones. On the other hand, in future UDNs with co-located sub-6GHz/mmWave deployment, the imbalance between uplink (UL) and downlink (DL) would urge the adoption of decoupling strategies. In that

H. Chergui and M. Benjillali are with the Communication Systems Department, INPT, Rabat, Morocco. [e-mail: chergui@ieee.org, benjillali@ieee.org].

M.-S. Alouini is with King Abdullah University of Science and Technology (KAUST), Computer, Electrical and Mathematical Science and Engineering Division (CEMSE), Thuwal 23955- 6900, Saudi Arabia. [e-mail: slim.alouini@kaust.edu.sa]. case, we may set a $K$-factor threshold to associate the UL to a LOS/OLOS gNB where, thanks to the minimal path-loss, the UE can reduce its transmit power allowing the reduction of UL signal to interference plus noise ratio (SINR) variance, which translates into more efficient and effective UL schedulers and performance gains [4]. Zooming out from the applications, a mathematical characterization of XLOS is yet to be established.

In this letter, we propose the XLOS service probability as a performance indicator, and start by introducing a broader definition of the concept thereof; accommodating the monitoring of both the UE serving and neighbor cells. Under the general framework of 5G large-scale parameters (LSPs) [5], we invoke a lognormal $K$-factor model for outdoor UEs [6] to conduct a closed-form analysis of the XLOS service probability in a 5G multi-tier heterogeneous network (HetNet). The obtained formula is including the different network parameters such as gNB density, height and antennas beamwidth, and expressed in terms of the multivariate Fox H-function [7, A.1], wherefore we provide a fast GPU-enabled MATLAB code. Finally, the asymptotic behavior highlighting the effect of different network and channel parameters on the availability of LOS conditions.

\section{SySTEM MOdeL}

Consider an outdoor $2 \mathrm{GHz}$ orthogonal frequency division multiple access (OFDMA)-based 5G [8] $N$-tiers UDN, where each cell class $n(n=1, \ldots, N)$ is modeled as a homogeneous Poisson point process (PPP) $\Phi_{n}$, and distinguished by its deployment density $\lambda_{n}$, maximum transmit power per resource element (RE) $P_{n}$, antennas height $h_{n}$ and beamwidth $\theta_{n}$. The corresponding channel is presenting a large scale fading, with constant path-loss exponent $\nu$ and lognormal shadowing $\mathcal{X}_{n}$ of mean $\mu_{n}$ and standard deviation $\sigma_{n}$. Assuming that UE locations follow an independent PPP $\Phi_{u}$ of density $\lambda_{u}$, the downlink analysis is performed at a typical UE located at the origin [9].

\section{A. Cell Monitoring Criteria}

As we are dealing with an outdoor context, we suppose that all tier's cells are open access (including femtocells). We also adopt a reference signal receive power (RSRP)-based cell selection, wherein each UE periodically monitors the collection of the $M$ strongest cells, dubbed here monitoring set, and ends up connecting to the best server. Since UE measurements rely on the long-term frequency-domain post-equalization receive power, small-scale fading variations do not impact cell selection/reselection and are not, therefore, reflected in the actual RSRP that reads

$$
P_{x_{n}}=P_{n} \mathcal{X}_{n}\left\|x_{n}\right\|^{-\nu}
$$

where $\left\|x_{n}\right\|^{-\nu}$ stands for the standard path-loss between a typical UE and an $n^{\text {th }}$-tier BS located at $x_{n} \in \Phi_{n}$. 


\section{B. K-Factor Model}

The $K$-factor-like all large scale parameters (LSPs)follows a lognormal distribution (cf. [5] and references therein). Without loss of generality, let us adopt the findings of [6], where we assume that the narrowband $K$-factor periodically measured by a UE at independent positions can be empirically modeled for the $n^{\text {th }}$-tier as,

$$
K_{x_{n}}=K_{n} \gamma_{n}\left\|x_{n}\right\|^{-\alpha},
$$

where $\alpha>0, K_{n}$ is the $K$-factor intercept defined as

$$
K_{n}=\left(h_{n} / h_{0}\right)^{\kappa_{1}}\left(\theta_{n} / \theta_{0}\right)^{\kappa_{2}} K_{0},
$$

with $\kappa_{1}>0, \kappa_{2}<0, K_{0}>0$, and $\gamma_{n}$ is an independent lognormal variable, whose decibel value is zero mean with a standard deviation $\sigma_{K}$. Accurate values of these model parameters can be obtained through a calibration process according to the target environment. New Jersey's measurement campaign in [6], for instance, yields $h_{0}=3 \mathrm{~m}, \theta_{0}=17^{\circ}, \alpha=0.5, \kappa_{1}=0.46$, $\kappa_{2}=-0.62, K_{0}=10$, and $\sigma_{K}=8 \mathrm{~dB}$. Note that this model involves also a seasonal factor $F_{s}$ that reflects the vegetation. For the sake of simplicity and without loss of generality, we consider the Summer's dense vegetation case $F_{s}=1$.

\section{Equivalent Formulation}

Since manipulating distances in PPPs is easier, let us transform the RSRP process (1) into a simple unit-power PPP $\widetilde{\Phi}_{n}$, where the strongest power would correspond to the nearest neighbor cell to the typical UE. By invoking the random displacement theorem [9, 1.3.9], [10, Corollary 3] shows that the two-dimensional (2D) process (1) is equivalent to another $2 \mathrm{D}$ process $P_{y_{n}}=\left\|y_{n}\right\|^{-\nu}$, such that $y_{n} \in \widetilde{\Phi}_{n}$ with density $\widetilde{\lambda}_{n}=$ $\lambda_{n} \Omega_{n}$, where $\Omega_{n}=P_{n}^{2 / \nu} \mathbf{E}\left[\mathcal{X}_{n}^{2 / \nu}\right]$ and the finite lognormal fractional moment $\mathbf{E}\left[\mathcal{X}_{n}^{2 / \nu}\right]=\exp \left[\frac{\ln 10}{5} \frac{\mu_{n}}{\nu}+\frac{1}{2}\left(\frac{\ln 10}{5} \frac{\sigma_{n}}{\nu}\right)^{2}\right]$. By means of the mapping theorem $[9,1.3 .11]$, the $K$-factor can also be re-expressed as

$$
K_{y_{n}}=K_{n} \gamma_{n} \Omega_{n}^{-\alpha / 2}\left\|y_{n}\right\|^{-\alpha}, y_{n} \in \widetilde{\Phi}_{n} .
$$

\section{Xlos Service Probability}

XLOS service probability in the vicinity of a UE, $P_{\mathrm{XLOS}}$, is defined as the probability that at least one cell in the monitoring set presents a $K$-factor higher than a threshold, say $K_{\text {th }}$, that can be fine-tuned depending on the target service, i.e.,

$$
P_{\mathrm{XLOS}}\left(K_{\mathrm{th}}\right) \triangleq \operatorname{Pr}\left[\bigcup_{m=1}^{M} K_{y_{n_{m}}}>K_{\mathrm{th}}, \mathbf{n} \in \mathcal{M}\right],
$$

where $\mathbf{n}=\left(n_{1}, \ldots, n_{M}\right)$ and $\mathcal{M}=\{1, \ldots, N\}^{M}$. In the sequel, we derive a closed-form expression for the XLOS service probability and study its asymptotic behavior.

\section{A. Closed-Form Analysis}

Using the total probability theorem as well as the independence between $\gamma_{n_{m}}, m=1, \ldots, M$, the definition (5) can be rewritten as

$$
\begin{aligned}
& P_{\mathrm{XLOS}}\left(K_{\mathrm{th}}\right)=1-\operatorname{Pr}\left[\bigcap_{m=1}^{M} K_{y_{n_{m}}} \leq K_{\mathrm{th}}, \mathbf{n} \in \mathcal{M}\right] \\
& =1-\sum_{\mathbf{n} \in \mathcal{M}} \operatorname{Pr}\left[y_{n_{m}} \in \widetilde{\Phi}_{n_{m}}, m=1, \ldots, M\right] \\
& \times \int_{0}^{z_{n_{2}}} \ldots \int_{0}^{z_{n_{M}}} \int_{0}^{+\infty} \prod_{m=1}^{M} \mathrm{CDF}_{\gamma_{n_{m}}}\left(\frac{K_{\mathrm{th}} \Omega_{n_{m}}^{\alpha / 2} z_{n_{m}}}{K_{n_{m}}} \mid z_{n_{m}}\right) \\
& \times f\left(z_{n_{1}}, \ldots, z_{n_{M}}\right) \mathrm{d} z_{n_{1}} \ldots \mathrm{d} z_{n_{M}},
\end{aligned}
$$

where $z_{n_{m}}=\left\|y_{n_{m}}\right\|^{\alpha}, f(\cdot)$ is the joint probability density function (PDF) whose variables verify $0 \leq z_{n_{1}} \leq z_{n_{2}} \leq \ldots \leq$ $z_{n_{M}}$, and CDF stands for the cumulative distribution function. Moreover, the independence between the homogeneous PPPs $\widetilde{\Phi}_{n_{m}}$ as well as the superposition theorem $[9,1.3 .3]$ imply that the sampling probability $\operatorname{Pr}\left[y_{n_{m}} \in \widetilde{\Phi}_{n_{m}}, m=1, \ldots, M\right]=$ $\prod_{m=1}^{M} \rho_{n_{m}}$, where $\rho_{n_{m}}=\widetilde{\lambda}_{n_{m}} / \lambda_{T}$ and $\lambda_{T}=\sum_{n=1}^{N} \widetilde{\lambda}_{n}$. To further develop (6), let us introduce the following new theorem.

Theorem 1 (Unified Expression for the Product of Lognormal $\left.\mathrm{CDFs}^{1}\right)$. Consider $M$ independent lognormal random variables $\gamma_{m}(m=1, \ldots, M)$, with mean $\mu_{m}(\mathrm{~dB})$ and standard deviation $\sigma_{m}(\mathrm{~dB})$. A unified expression for the product of their individual CDFs-that is equal to their joint CDF $\mathrm{CDF}_{\gamma_{1}, \ldots, \gamma_{M}}\left(\gamma_{\mathrm{th}, 1}, \ldots, \gamma_{\mathrm{th}, M}\right)$-is given by

$$
\prod_{m=1}^{M} \operatorname{CDF}_{\gamma_{m}}\left(\gamma_{\mathrm{th}, m}\right)=\frac{1}{\pi^{M / 2}} \sum_{l=1}^{L} w_{l} \prod_{m=1}^{M} \mathrm{H}_{1,1}^{0,1}\left[\frac{\gamma_{\mathrm{th}, m}}{\omega_{l, m}} \mid \begin{array}{c}
(1,1) \\
(0,1)
\end{array}\right],
$$

where $\omega_{l, m}=10^{\left(\sqrt{2} \sigma_{m} u_{l, m}+\mu_{m}\right) / 10}$ for $l \in\{1, \ldots, L\}, w_{l}$ and $\left(u_{l, 1}, \ldots, u_{l, M}\right)$ are respectively the weight and the $M$ abscissas of the $L^{\text {th }}$-order $M$-dimensional Gaussian weight Stroud monomial cubature [12], with $\sum_{l=1}^{L} w_{l}=\pi^{M / 2}$ and

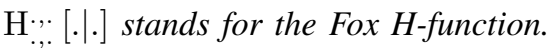

\section{Proof: cf. Appendix A.}

On the other hand, an explicit expression of the joint PDF $f(\cdot)$ can be obtained via the following corollary.

Corollary 1 (of Theorem [13, Appendix]). In a multitier random network modeled in terms of $N$ independent PPPs $\widetilde{\Phi}_{n}(n=1, \ldots, N)$ with densities $\widetilde{\lambda}_{n}$, let $z_{m}=$ $r_{m}^{\alpha}(m=1, \ldots, M)$, such that $r_{m}$ is the distance of the $m^{\text {th }}$ neighbor with respect to a certain origin. The joint PDF of $z_{1}, \ldots, z_{M}$ unconditionally to $\left\{\widetilde{\Phi}_{n}\right\}$ reads

$$
f\left(z_{1}, \ldots, z_{M}\right)=\left(\frac{2 \pi \lambda_{T}}{\alpha}\right)^{M} e^{-\pi \lambda_{T} z_{M}^{2 / \alpha}} \prod_{m=1}^{M} z_{m}^{2 / \alpha-1},
$$

where $\lambda_{T}=\sum_{n=1}^{N} \tilde{\lambda}_{n}$.

Proof: cf. Appendix B.

By making use of the aforementioned sampling probability as well as Theorem 1 and Corollary 1, the XLOS service probability (6) can be rewritten after some algebraic manipulations as,

$$
P_{\mathrm{XLOS}}\left(K_{\mathrm{th}}\right)=1-\left(\frac{2 \sqrt{\pi}}{\alpha}\right)^{M} \sum_{\mathbf{n} \in \mathcal{M}} \prod_{m=1}^{M} \widetilde{\lambda}_{n_{m}} \sum_{l=1}^{L} w_{l} \times I_{1},
$$

\footnotetext{
${ }^{1}$ This theorem can be viewed as a generalization of the well-established Gauss-Hermite representations of the lognormal PDF and CDF (see e.g., [11]).
} 


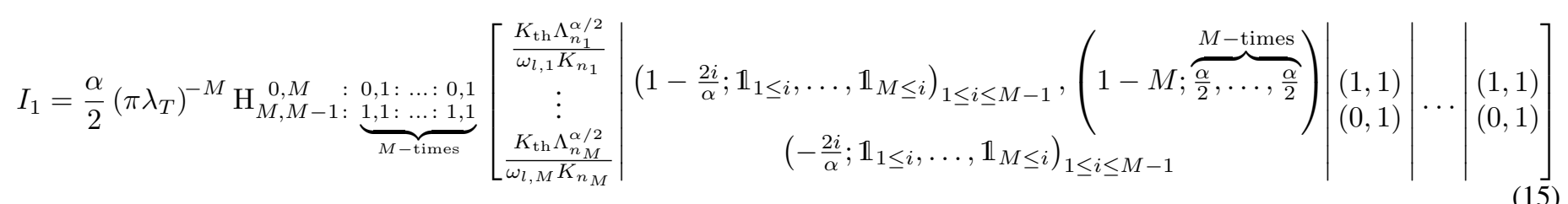

where the multidimensional integral $I_{1}$ is expressed as

$$
\begin{gathered}
I_{1}=\int_{0}^{z_{n_{2}}} \ldots \int_{0}^{z_{n_{M}}} \int_{0}^{+\infty} \prod_{m=1}^{M} z_{n_{m}}^{2 / \alpha-1} \mathrm{H}_{1,1}^{0,1}\left[\frac{K_{\mathrm{th}} \Omega_{n_{m}}^{\alpha / 2} z_{n_{m}}}{\omega_{l, m} K_{n_{m}}} \mid \begin{array}{c}
(1,1) \\
(0,1)
\end{array}\right] \\
\times e^{-\pi \lambda_{T} z_{n_{M}}^{2 / \alpha} \mathrm{d} z_{n_{1}} \ldots \mathrm{d} z_{n_{M}} .}
\end{gathered}
$$

To derive a closed-form solution for (10), let us recall the representation of the involved Fox $\mathrm{H}$-functions in terms of Mellin-Barnes integrals [7, Eq. (1.1.1)], i.e,

$$
\mathrm{H}_{1,1}^{0,1}\left[\begin{array}{c|c}
z & (1,1) \\
(0,1)
\end{array}\right]=\frac{1}{2 \pi j} \int_{\mathcal{C}_{m}} \phi\left(\zeta_{m}\right) z^{\zeta_{m}} \mathrm{~d} \zeta_{m}
$$

where $\phi\left(\zeta_{m}\right)=\Gamma\left(\zeta_{m}\right) / \Gamma\left(1+\zeta_{m}\right)$, and contours $\mathcal{C}_{m}(m=1, \ldots, M)$ are defined such that $\operatorname{Re}\left(\zeta_{m}\right)>0$; the highest pole on the left. Combining (11) with (10) and interchanging the order of the real and contour integrals-which is permissible given the absolute convergence of the involved integrals-we obtain,

$$
\begin{aligned}
I_{1} & =\left(\frac{1}{2 \pi j}\right)^{M} \int_{\mathcal{C}_{1}} \ldots \int_{\mathcal{C}_{M}} \Psi\left(\zeta_{1}, \ldots, \zeta_{M}\right) \\
& \times \prod_{m=1}^{M} \phi\left(\zeta_{m}\right)\left(\frac{K_{\mathrm{th}} \Omega_{n_{m}}^{\alpha / 2}}{\omega_{l, m} K_{n_{m}}}\right)^{\zeta_{m}} \mathrm{~d} \zeta_{1} \ldots \mathrm{d} \zeta_{M},
\end{aligned}
$$

with the multivariate term $\Psi$ given by

$$
\begin{aligned}
\Psi\left(\zeta_{1}, \ldots, \zeta_{M}\right) & =\int_{0}^{z_{n_{2}}} \ldots \int_{0}^{z_{n_{M}}} \int_{0}^{+\infty} e^{-\pi \lambda_{T} z_{n_{M}}^{2 / \alpha}} \\
& \times \prod_{m=1}^{M} z_{n_{m}}^{2 / \alpha+\zeta_{m}-1} \mathrm{~d} z_{n_{1}} \ldots \mathrm{d} z_{n_{M}} .
\end{aligned}
$$

Given that $\alpha \in \mathbb{R}^{+*}$ and $\operatorname{Re}\left(\zeta_{m}\right)>0$, and using the identity $1 / a=\Gamma(a) / \Gamma(1+a)$, the iterated integrals with respect to $z_{n_{1}}, \ldots, z_{n_{M-1}}$ in (13) can be successively resolved by induction. The resulting integral relating to $z_{n_{M}}$ is then obtained using [14, Eq. (3.478.1)], which leads to

$$
\begin{gathered}
\Psi\left(\zeta_{1}, \ldots, \zeta_{M}\right)=\frac{\alpha}{2}\left(\pi \lambda_{T}\right)^{-\left(M+\frac{\alpha}{2} \sum_{m=1}^{M} \zeta_{m}\right)} \\
\times \Gamma\left(M+\frac{\alpha}{2} \sum_{m=1}^{M} \zeta_{m}\right) \prod_{i=1}^{M-1} \frac{\Gamma\left(\frac{2 i}{\alpha}+\sum_{m=1}^{M} \mathbb{1}_{m \leq i} \zeta_{m}\right)}{\Gamma\left(1+\frac{2 i}{\alpha}+\sum_{m=1}^{M} \mathbb{1}_{m \leq i} \zeta_{m}\right)} .
\end{gathered}
$$

By plugging (14) into (12), we recognize that integral $I_{1}$ can be re-expressed in terms of the multivariate Fox H-function [7, A.1] as given by (15) on top of this page, where parameter $\Lambda_{n_{m}} \triangleq \Omega_{n_{m}} / \pi \lambda_{T}$ is encompassing network density, power and shadowing effects. Finally, a closed-form expression for $P_{\mathrm{XLOS}}$ is deduced by substituting (15) in (9).

\section{B. Asymptotic Behavior}

As depicted in Table I, the two asymptotic regimes of the ratio $K_{\mathrm{th}} \Lambda_{n_{m}}^{\alpha / 2} / \omega_{l, m} K_{n_{m}}$ (in $W^{\alpha / \nu} m^{2}$ ) reflect many practical scenarios, wherefore it is interesting to establish the corresponding XLOS service probability expressions; denoted $\bar{P}_{\text {XLOS }}$ in the sequel. Let $\mathcal{H}$ stand for the multivariate Fox H-function in (15) where

$$
\mathcal{H}=\left(\frac{1}{2 \pi j}\right)^{M} \int_{\mathcal{C}_{1}} \ldots \int_{\mathcal{C}_{M}} F\left(\zeta_{1}, \ldots, \zeta_{M}\right) \mathrm{d} \zeta_{1} \ldots \mathrm{d} \zeta_{M} .
$$

In view of the series representations of the monovariate Fox $\mathrm{H}$-function [15, Theorem 1.2] (while noticing the inverted definition of the $\mathrm{H}$-function therein), an asymptotic expression of (15) is obtained as follows.

Low ratio regime: Since the integrand $F$ has no poles on the right of the $M$ individual contours in (16), [15, Eq. (1.2.23)] implies that $\mathcal{H} \simeq 0$, and thereby $\bar{P}_{\mathrm{XLOS}}=1$.

High ratio regime: By applying [15, Eq. (1.2.22)] to the $M$ individual contour integrals, an approximation of $\mathcal{H}$ is given in terms of the residues of $F$ as

$$
\begin{aligned}
& \mathcal{H} \simeq \operatorname{Res}[F,(0, \ldots, 0)]+\operatorname{Res}\left[F,\left(-\frac{2}{\alpha}, 0, \ldots, 0\right)\right] \\
& \simeq \lim _{\zeta_{M} \rightarrow 0} \ldots \lim _{\zeta_{1} \rightarrow 0} \prod_{m=1}^{M} \zeta_{m} F\left(\zeta_{1}, \ldots, \zeta_{M}\right) \\
&+\lim _{\zeta_{M} \rightarrow 0} \ldots \lim _{\zeta_{2} \rightarrow 0} \lim _{\zeta_{1} \rightarrow-\frac{2}{\alpha}}\left(\zeta_{1}+\frac{2}{\alpha}\right) \prod_{m=2}^{M} \zeta_{m} F\left(\zeta_{1}, \ldots, \zeta_{M}\right),
\end{aligned}
$$

\begin{tabular}{|c|c|c|}
\hline Case & Practical Scenarios & $\bar{P}_{\mathrm{XLOS}}$ \\
\hline$\frac{K_{\mathrm{th}} \Lambda_{n_{m}}^{\alpha / 2}}{\omega_{l, m} K_{n_{m}}} \rightarrow 0$ & $\begin{array}{l}\text { - Lightly shadowed clutter (low } \\
\Omega_{n_{m}} \text {,) } \\
\text { - Fair LOS quality in a UDN with } \\
\text { high power and narrow-beam an- } \\
\text { tennas (i.e., high } \lambda_{T} \text { and } K_{n_{m}} \text { ). }\end{array}$ & 1 \\
\hline$\frac{K_{\mathrm{th}} \Lambda_{n_{m}}^{\alpha / 2}}{\omega_{l, m} K_{n_{m}}} \rightarrow+\infty$ & $\begin{array}{l}\text { - Severely shadowed clutter (high } \\
\Omega_{n_{m}} \text { ), } \\
\text { - Fair LOS quality in a low density } \\
\text { HetNet with low power and large } \\
\text { beamwidth antennas (i.e., low } \lambda_{T} \\
\text { and } K_{n_{m}} \text { ). }\end{array}$ & $\begin{array}{l}\text { Equation } \\
\text { (19) }\end{array}$ \\
\hline
\end{tabular}

which evaluates to

$$
\mathcal{H} \simeq\left(\frac{\alpha}{2}\right)^{M-1}\left[1-\left(\frac{K_{\mathrm{th}} \Lambda_{n_{1}}^{\alpha / 2}}{\omega_{l, 1} K_{n_{1}}}\right)^{-2 / \alpha}\right] .
$$

Finally, combining (9), (15) and (18), as well as recalling that $\sum_{l=1}^{L} w_{l}=\pi^{M / 2}$, we obtain after some algebraic manipulations

$$
\bar{P}_{\mathrm{XLOS}}=\frac{1}{\pi^{M / 2-1}} \sum_{\mathbf{n} \in \mathcal{M}} \frac{\lambda_{T}}{\Omega_{n_{1}}} \prod_{m=1}^{M} \rho_{n_{m}} \sum_{l=1}^{L} w_{l}\left(\frac{\omega_{l, 1} K_{n_{1}}}{K_{\mathrm{th}}}\right)^{2 / \alpha} \text {. }
$$

Table I

XLOS SERVICE PROBABILITY ASYMPTOTIC EXPRESSIONS 


\section{Numerical Results AND Mathematical Software}

To validate our theoretical findings, we conduct Monte-Carlo simulations for three practical scenarios as depicted in Table II, and we adopt New Jersey's calibration presented in II-B with $\sigma_{K}=3 \mathrm{~dB}$. The analytical expressions are evaluated via a degree-11 Stroud cubature for which $L=\left(4 M^{5}-20 M^{4}+\right.$ $\left.140 M^{3}-130 M^{2}+96 M+15\right) / 15$. To that end, we make use of Stenger's tabulations [16] to update the MATLAB code in [17]. Moreover, we introduce in [18] an efficient GPU-oriented MATLAB routine to calculate the multivariate Fox H-function.

Fig. 1 shows that, in a UDN with light shadowing, LOS links are easily established (e.g., $K_{\text {th }}=12 \mathrm{~dB}$ is obtained with probability 1). Conversely, the low density network (LDN) scenario unfolds in NLOS situations with non-negligible probability (e.g., $K<-5 \mathrm{~dB}$ with probability 0.5 ). By considering the neighboring cells $(M=2,3)$ in the HetNet (Macro/Femto) case for instance, we remark that a substantial increase of the XLOS probability is achieved only in the non-asymptotic regime. Indeed, a high $K_{\mathrm{th}}$ requirement can be fulfilled merely by the serving cell, since the $K$-factors of neighbor cells become limited by the corresponding path-losses as implied by (2).

Table II

NETWORK AND TRANSMISSION SETTINGS

\begin{tabular}{|l|c|c|c|c|c|c|}
\hline Case & $N$ & $\lambda_{n}$ & $P_{n}(\mathrm{dBm})$ & $\theta_{n}\left({ }^{\circ}\right)$ & $h_{n}(\mathrm{~m})$ & $\sigma_{n}(\mathrm{~dB})$ \\
\hline \hline UDN & 1 & $3 \times 10^{-2}$ & 5.2 & 45 & 15 & 5 \\
\hline HetNet & 2 & $10^{-5}, 5 \times 10^{-4}$ & $15.2,-4.8$ & 39,180 & 25,10 & 8,4 \\
\hline LDN & 1 & $3 \times 10^{-8}$ & 5.2 & 65 & 30 & 5 \\
\hline
\end{tabular}

\section{CONCLUSion}

In this letter, we have introduced the XLOS service probability as a new $K$-factor-based performance indicator, and provided its analytical and asymptotic expressions that unveil the effect of the variation of 5G network and transmission parameters on the gradual visibility condition of radio links. By tweaking a $K$ factor threshold $K_{\mathrm{th}}$, the XLOS metric can be used by network optimization algorithms as the probability of e.g., reconfiguring the uplink in a LOS/OLOS gNB. As a perspective, the adopted $K$-factor model from [6] can be extended to the vehicular case in future works.

\section{APPENDIX A \\ PROOF OF THEOREM 1}

First, by making a simple variable change, the product of lognormal PDFs $p_{\gamma_{m}}, p=\prod_{m=1}^{M} p_{\gamma_{m}}$, can be reformulated as

$$
p=\frac{1}{\pi^{M / 2}} \int_{\mathbb{R}^{M}} e^{-\left(u_{1}^{2}+\ldots+u_{M}^{2}\right)} Q\left(u_{1}, \ldots, u_{M}\right) \mathrm{d} u_{1} \ldots \mathrm{d} u_{M},
$$

where $Q\left(u_{1}, \ldots, u_{M}\right)=\prod_{m=1}^{M} \delta\left(\gamma_{m}-10^{\left(\sqrt{2} \sigma_{m} u_{m}+\mu_{m}\right) / 10}\right)$. By applying the Gaussian-weight Stroud monomial cubature [12] to (20), and recalling that $\delta\left(\gamma_{m}-a\right)=\mathrm{H}_{0,0}^{0,0}\left[\frac{\gamma_{m}}{a} \mid-\right]$, we get

$$
p=\frac{1}{\pi^{M / 2}} \sum_{l=1}^{L} w_{l} \prod_{m=1}^{M} \mathrm{H}_{0,0}^{0,0}\left[\frac{\gamma_{m}}{\omega_{l, m}} \mid-\right],
$$

with $w_{l}$ and $\left(u_{l, 1}, \ldots, u_{l, M}\right)$ are respectively the $l^{\text {th }}$ weight and abscissas of the $M$-dimensional cubature, and $\omega_{l, m}=$ $10^{\left(\sqrt{2} \sigma_{m} u_{l, m}+\mu_{m}\right) / 10}$. Finally, by invoking [7, Eq. (2.53)], the integration of (21) with respect to $\gamma_{m}$ from 0 to $\gamma_{\mathrm{th}, \mathrm{m}}(m=$ $1, \ldots, M)$ leads to (7).

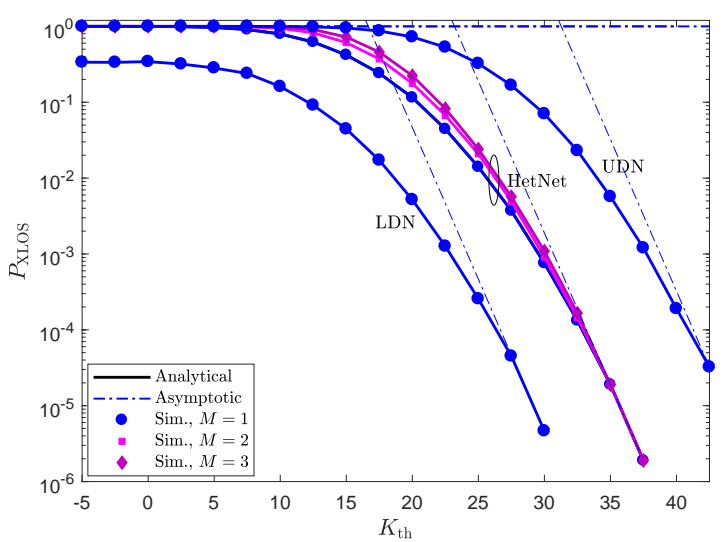

Figure 1. XLOS probability versus $K_{\mathrm{th}}$ for UDN, HetNet (Macro/Femto) and LDN. Path-loss exponent $\nu=3$ and shadowing mean $\mu_{n}=0$ for all tiers $n=1, \ldots, N$.

\section{APPENDIX B \\ PROOF OF COROLLARY 1}

It immediately follows from applying the superposition theorem $[9,1.3 .3]$ to the equivalent PPP $\Phi_{T}=\bigcup_{n=1}^{N} \widetilde{\Phi}_{n}$, and performing a PDF transformation to the joint distance distribution of the first $M$ neighbors given by theorem [13, Appendix].

\section{REFERENCES}

[1] S. Sun et al., "MIMO for millimeter wave wireless communications: Beamforming, spatial multiplexing, or both?," IEEE Comm. Mag., vol 52, no. 12, pp. 110-121, Dec. 2014.

[2] L. Bernado et al., "Time- and frequency-varying $K$-factor of nonstationary vehicular channels for safety relevant scenarios" IEEE Trans. on Intell. Transp. Sys., vol. 16, no. 2, pp. 1007-1017, Apr. 2015

[3] N. Garcia et al., "Direct localization for massive MIMO," IEEE Trans. Sig. Proc., vol. 65, no. 10, pp 2475-2487, May 2017.

[4] H. Elshaer et al., "Decoupled uplink and downlink access in heterogeneous networks," Chapter 8 in $5 G$ Wireless Technologies, IET Digital Library, 2017.

[5] 3GPP, "Study on channel model for frequencies from 0.5 to $100 \mathrm{GHz}$ (Release 14)," TR 38.901, May. 2017.

[6] L. J. Greenstein et al., "Ricean $K$-factors in narrow-band fixed wireless channels: Theory, experiments, and statistical models,"IEEE Trans. Veh. Tech., vol. 58, no. 8, pp. 4000-4012, Oct. 2009.

[7] A. M. Mathai, R. K. Saxena, and H. J. Haubold, The H-Function: Theory and Applications, Springer, New York, 2010.

[8] 3GPP, "NR: Physical channels and modulation (Release 15)," TS 38.211, Sep. 2017

[9] F. Baccelli and B. Blaszczyszyn, Stochastic Geometry and Wireless Networks, Volume I-Theory. NOW: Foundations and Trends in Networking, 2009.

[10] P. Madhusudhanan et al., "Downlink performance analysis for a generalized shotgun cellular systems," 2012. [Online]. Available: arxiv.org/abs/1002.3943.

[11] F. Yilmaz and M.-S. Alouini, "A novel unified expression for the capacity and bit error probability of wireless communication systems over generalized fading channels," IEEE Trans. Commun., vol. 60, no. 7, pp. 1862-1876, July 2012.

[12] A. H. Stroud, Approximate Calculation of Multiple Integrals, Englewood Cliffs, N.J.:Prentice-Hall, 1971.

[13] H. R. Thompson, "Distribution of distance to nth neighbour in a population of randomly distributed individuals," Ecology, vol. 37, no. 2, pp. 391-394, Apr. 1956.

[14] I. S. Gradshteyn and I. M. Ryzhik, Table of Integrals, Series, and Products, 7th ed., Academic Press, 2007.

[15] A. Kilbas and M. Saigo, H-Transforms: Theory and Applications. Analytical Methods and Special Functions, Chapman \& Hall/CRC, Boca Raton-London-New York-Washington, D.C., 2004.

[16] F. Stenger, "Tabulation of certain fully symmetric numerical integration formulas of degree 7, 9 and 11," Mathematics of Computation, vol. 25, no. 116, pp. 935 and S58-S125, Oct. 1971.

[17] J. Burkardt, "Stroud numerical integration in $M$ dimensions," 2010. [Online]. Available: people.sc.fsu.edu/ jburkardt/m_src/stroud/stroud.html.

[18] H. Chergui, M. Benjillali and M.-S. Alouini, "GPU-Enabled Multivariate Fox H-function Code," Zenodo, Aug. 2018. DOI: 10.5281/zenodo. 1400403 . 